\title{
On Joint Power Allocation and Multipath Routing in Femto-Relay Networks
}

\author{
Sahar Hoteit, Pierre Duhamel, Samson Lasaulce \\ Laboratoire des Signaux et Systèmes (L2S, UMR CNRS 8506), CNRS-CentraleSupélec-Université Paris-Sud, 91192 \\ Gif-sur-Yvette, France (e-mails: sahar.hoteit@1ss.supelec.fr, pierre.duhamel@1ss.supelec.fr, samson.lasaulce@1ss.supelec.fr)
}

\begin{abstract}
Transmit power allocation techniques are very important to manage interference in small-cell networks. While available power allocation algorithms in the literature rely on a predefined routing protocol, we propose in this paper a power-efficient two-step algorithm that allows power allocation and routing to be performed jointly in femto-relay networks. First, we propose an interference-based partitioning method to cluster the femto-relays, then we adopt an iterative and distributed algorithm, inspired from game theory, for efficient transmit power allocation. We show that the corresponding power allocation game possesses a pure Nash equilibrium which is reached by the proposed algorithm within a number of iterations per femto-relay which can be as small as 1 . Moreover, we show that our approach grants significant improvements in terms of power consumption, and permits the total consumed power to be divided by about 6 and 3 when respectively compared to the direct transmission and shortest path techniques.
\end{abstract}

\section{INTRODUCTION}

Femto-relays have recently appeared as a novel solution for next generation small cell problems [1]. They provide a dualbackhaul connectivity to the core network for both registered and unregistered users: the internet-based connectivity as well as the relay-based operating on the spectrum owned by the wireless carrier [2]. Working as open-access small cells, they permit to increase the overall system capacity and to offload traffic from existing macro-cellular networks [3]. This holds for downlink and uplink communications since a macro user who has a weak direct link with the corresponding macrocell and who is located at the vicinity of an open-access femtorelay, can have a better signal quality and throughput if receiving or transmitting the data through the neighboring openaccess femto-relays. He can obtain energy savings if using the multi-hop transmission through the open-access femto-relay network instead of the direct link with the macrocell.

Femto-relays intend to improve over previous solutions based on femto-cells, which have faced several major challenges [4] [5], especially in terms of cross-tier (i.e., the interference that occurs between the macrocells and the femtocells sharing the same frequency band), as well as in terms of backhaul bottleneck. In fact, the crucial problem of the crosstier interference can be mitigated by serving the macrocell users generating the largest interference via a relay-based backhaul through femto-relays, provided that these users can find an appropriate path. Moreover, the key innovation of these networks that consists of using multiple backhauls to access the core network further improves users quality of service. There are however technical challenges with femto-relays deployment. The major issue remains the co-tier interference that occurs between neighboring femtocells. This problem is often addressed via a certain coordination through a centralized management entity [2].

In this context, transmit power allocation technique appears to the forefront as one of the most conventional practices to mitigate the co-tier and to increase system capacity [6]. Indeed, the transmit power level of a femto-relay affects its coverage range and the amount of interference it generates in the network. Although higher transmit power can provide wider coverage and better signal quality, it can, at the same time, cause tremendous interference to other surrounding users of the neighboring femtocells.

Different power allocation techniques have been recently considered to mitigate the various types of interference in two-tier networks with co-channel deployed femtocells. For instance, [7] proposes a two-step approach based on a joint Voronoi diagram and a game theory-based power control scheme. Moreover, authors in [9] propose a distributed power allocation algorithm based on a Stackelberg model for spectrum sharing in femtocell networks in which the total capacity is maximized and femtocell users are priced for causing interference to the macrocells. In [10], a distributed power and subchannel allocation technique is proposed for co-channel femtocell deployments using a non-cooperative game modeling in which a Nash equilibrium is obtained based on a time-sharing subchannel allocation. However the constraint on the maximum femtocell transmit power is not considered in the game. Similarly, authors in [11] propose two interference mitigation strategies in which femtocell users adjust the maximum transmit power, using an open-loop and a closed-loop technique. In contrast to other works, authors in [12] focus only on the co-tier interference, and propose a distributed power allocation scheme for closed-access femtocell networks. They model the problem as a supermodular game where the players are the femtocell access points, whose actions are their transmitted powers and whose utilities are their capacities. Authors in [13] formulate the power control problem as a generalized Nash equilibrium game, they minimize the transmit power under quality of service constraints. Their formulation is applied on a fixed routing, and they do not use bidirectional communications. In contrast to these works, this paper is concerned with open access femto relay networks and with co-tier interference. Our objective is to ensure a joint power allocation and multi-path routing in a femto-relay network consisting of bi-directional communications. In our setting, all nodes share the same bandwidth and we allow the communications to be 
split via several routes, provided that it corresponds to a better solution. The problem is solved using a two-step approach: the first is an interference-based partitioning of the network, while the second consists of an iterative and distributed power allocation algorithm, based on the sequential best response dynamics and modeled using game theory. We show that the proposed approach admits a Nash equilibrium and converges within a finite number of iterations. Moreover, comparing our algorithm with classical routing techniques from the literature, we show that our approach grants important improvements in terms of power reduction. The paper is organized as follows. Section II analytically introduces the context of our work and formulate the problem using a centralized optimization problem. Section III describes the proposed approach, followed by a presentation of simulation results in Section IV. Finally, Section V concludes this paper.

\section{Context and Problem Formulation}

Consider a network composed of a macrocell with several femto-relays (FRs) that operate as open-access small cells. We assume, as in [14], that femto-relays operate using the time division duplex (TDD) mode that uses a single frequency band for both transmit and receive, then it shares that band by assigning alternating time slots to transmit and receive operations. All femto-relays are assumed to use the same frequency band. We model the case of a high-density network where the demand of some macrocell users would generate tremendous interference, and impact other communications. Our objective is thus to transmit the traffic of these users through femto-relays with the least consumed power, while ensuring that no data loss occurs in the network. As shown in [2], the co-tier interference in femto-relay networks often requires a certain coordination through a centralized management entity. Therefore, we first present the corresponding centralized optimization problem, following the approach in [8] which was obtained in another context, then we present our two-step algorithm for the joint power allocation and multipath routing.

\section{A. Notations}

Let $\mathcal{N}$ be the set of femto-relays in the network whose cardinality is $n=|\mathcal{N}|, P_{i}$ is the transmit power of the femtorelay $N_{i} \in \mathcal{N}$ that is equal to the sum of transmit power of the outgoing links from $N_{i}$ (i.e., $P_{i}=\sum_{j} P_{i j}$, where $P_{i j}$ represents the transmit power of the link from $N_{i}$ to $N_{j}$, denoted by $\ell_{i, j}$ ). $P_{i}$ is upper bounded by a maximum transmit power $P_{\max }^{i}$. Also, let $r_{i j}$ be the rate of link $\ell_{i, j}, c_{i j}$ be its capacity (i.e., the theoretical highest data rate that can be sent through this link) such as:

$$
r_{i j} \leq c_{i j}
$$

The normalized link capacity is given by the Shannon theorem:

$$
c_{i j}=\log _{2}\left(1+\mathrm{SINR}_{i j}\right)
$$

where $\mathrm{SINR}_{i j}$ represents the signal to interference-plus-noise ratio of link $\ell_{i, j}$ at the receiver $j$. This SINR is given by:

$$
\mathrm{SINR}_{i j}=\frac{L_{i j} F_{i j} P_{i j}}{\sigma_{j}^{2}+\sum_{k=1 ; k \neq i, j}^{n} L_{k j} F_{k j} P_{k}}
$$

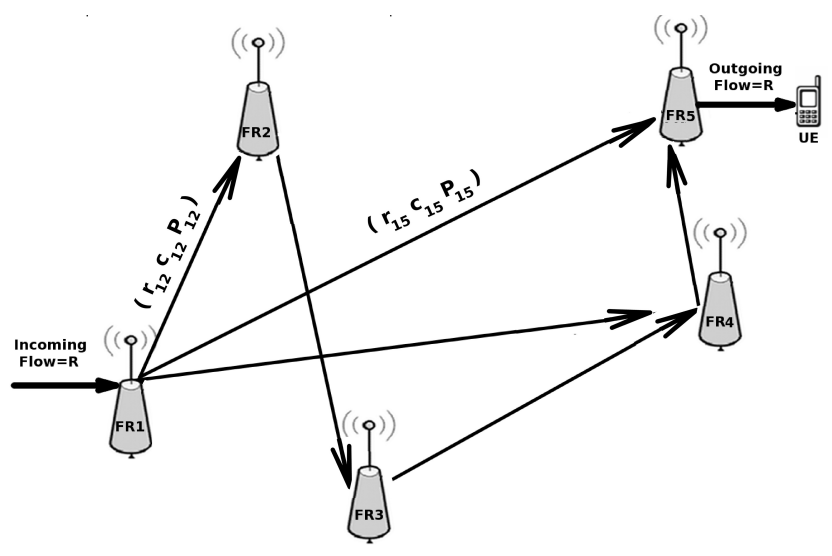

Fig. 1. A femto-relay network where the arrows represent the transmissions between different nodes (bi-directional communications). Each link is characterized by the triplet (rate, capacity, Power).

where:

- $L_{i j}$ represents the path loss at receiver $N_{j}$ (in the simulations, as in [4], we adopt the path loss model given in the A1 scenario for indoor small office and residential of WINNER [15]).

- $F_{i j}$ represents the effects by fast fading modeled using a Rayleigh fast fading channel that follows a negative exponential distribution [16], and

- $\sigma_{j}^{2}$ represents the background noise power at receiver $j$ (the simulations use an additive white Gaussian noise).

For simplicity, we refer in the following to the product of the path loss and the effects of fast fading as $G_{i j}=L_{i j} F_{i j}$ that represents the path gain of link $\ell_{i j}$. For example, consider the situation depicted in Fig. 1, with five femto-relays that have to transmit the incoming flow $R$ among the different links such as there is no data loss (i.e., the outgoing flow is equal to the incoming flow). The triplet $\left(r_{i j}, c_{i j}, P_{i j}\right)$ represents the rate, the capacity and the power of the link between $N_{i}$ and $N_{j}$. Note that the communications in the network are bidirectional, but the links are asymmetric i.e. the characteristics of link $\ell_{i j}$ are different of those of link $\ell_{j i}$, since interference depends on the location of nodes $i$ and $j$.

\section{B. Related centralized optimization problem}

In this section, we formulate the joint power allocation and multi-path routing problem as a centralized single decisionmaker optimization problem whose objective is to find the minimal transmit power that ensures the delivery of a flow at a given bit rate. This formulation is useful in the sense that it permits to clearly identify the difficulties in terms of optimization and the complexity especially in high-density network, and it can be considered as an intermediary step towards our proposed approach. This centralized setting would correspond to the case where a centralized management entity ensures the coordination between the different femtorelays [2], the centralized node might be the macrocell for example. Using (1), (2) and (3), we can express the transmit power of each $N_{i} \in \mathcal{N}$ by: 


$$
\begin{aligned}
P_{i} & =\sum_{\substack{1 \leq j \leq n \\
j \neq i}} P_{i j} \\
& =\sum_{\substack{1 \leq j \leq n \\
j \neq i}} \frac{\left(2^{c_{i j}}-1\right)\left(\sigma_{j}^{2}+\sum_{\substack{k=1 \\
k \neq i, j}}^{n} G_{k j} P_{k}\right)}{G_{i j}} \\
& \geq \sum_{\substack{1 \leq j \leq n \\
j \neq i}} \frac{\left(2^{r_{i j}}-1\right)\left(\sigma_{j}^{2}+\sum_{\substack{k=1 \\
k \neq i, j}}^{n} G_{k j} P_{k}\right)}{G_{i j}}
\end{aligned}
$$

This can also be written in matrix form as: $\mathcal{A}(\overrightarrow{\mathbf{r}}) \times \mathcal{P}(\overrightarrow{\mathbf{r}}) \geq$ $\mathcal{B}(\overrightarrow{\mathbf{r}})$ where:

- $\overrightarrow{\mathbf{r}}$ is the rate vector, obtained by vectorizing ${ }^{1}$ the transpose of an $n \times n$ hollow matrix ${ }^{2} \mathcal{R}$ whose $(i, j)^{t h}$ entry represents the rate of link $\ell_{i j}$ :

$$
\mathcal{R}=\left(\begin{array}{cccc}
0 & r_{12} & \cdots & r_{1 n} \\
r_{21} & 0 & \cdots & r_{2 n} \\
\vdots & \vdots & \ddots & \vdots \\
r_{n 1} & r_{n 2} & \cdots & 0
\end{array}\right)
$$

Henceforth, the rate vector is given by: $\overrightarrow{\mathbf{r}}=\left(\operatorname{vec}\left(\mathcal{R}^{T}\right)\right)^{T}$ $=\left(0, r_{12}, \cdots, r_{1 n}, r_{21}, 0, \cdots, r_{2 n}, \cdots, r_{n 1}, r_{n 2}, \cdots, 0\right)$

- The transmit power vector is given by: $\mathcal{P}(\overrightarrow{\mathbf{r}})=\left(\begin{array}{c}P_{1}(\overrightarrow{\mathbf{r}}) \\ P_{2}(\overrightarrow{\mathbf{r}}) \\ \vdots \\ P_{n}(\overrightarrow{\mathbf{r}})\end{array}\right)$ $\boldsymbol{B}(\overrightarrow{\mathbf{r}})=\left(\begin{array}{c}\sum_{\substack{1 \leq j \leq n \\ j \neq 1}} \frac{\left(2^{r_{1 j}}-1\right) \sigma_{j}^{2}}{G_{1 j}} \\ \sum_{\substack{1 \leq j \leq n \\ j \neq 2}} \frac{\left(2^{r_{2 j}}-1\right) \sigma_{j}^{2}}{G_{2 j}} \\ \vdots \\ \sum_{\substack{1 \leq j \leq n \\ j \neq n}} \frac{\left(2^{r_{n j}}-1\right) \sigma_{j}^{2}}{G_{n j}}\end{array}\right)$, and

- $\mathcal{A}(\overrightarrow{\mathbf{r}})$ is equal to:

$$
\left(\begin{array}{cccc}
1 & \sum_{\substack{1 \leq j \leq n \\
j \neq 1}} \frac{\left(1-2^{r_{1 j}}\right) G_{2 j}}{G_{1 j}} & \ldots \sum_{\substack{1 \leq j \leq n \\
j \neq 1}} \frac{\left(1-2^{r_{1 j}}\right) G_{n j}}{G_{1 j}} \\
\sum_{\substack{1 \leq j \leq n \\
j \neq 2}} \frac{\left(1-2^{r_{2 j}}\right) G_{1 j}}{G_{2 j}} & 1 & \ldots \sum_{\substack{1 \leq j \leq n \\
j \neq 2}} \frac{\left(1-2^{r_{2 j}}\right) G_{n j}}{G_{2 j}} \\
\vdots & & \ddots & \vdots \\
\sum_{\substack{1 \leq j \leq n \\
j \neq n}} \frac{\left(1-2^{r_{n j}}\right) G_{1 j}}{G_{n j}} \sum_{\substack{1 \leq j \leq n \\
j \neq n}} \frac{\left(1-2^{r_{n j}}\right) G_{2 j}}{G_{n j}} & \cdots & 1
\end{array}\right)
$$

For the sake of clarity, we refer thereafter to $\mathcal{A}(\overrightarrow{\mathbf{r}})$ and $\mathcal{B}(\overrightarrow{\mathbf{r}})$ as $\mathcal{A}_{\mathbf{r}}$ and $\mathcal{B}_{\mathbf{r}}$, respectively.

As our objective in this paper is to minimize the femtorelays transmit powers while ensuring the delivery of the

\footnotetext{
${ }^{1}$ The vectorization of a matrix is a linear transformation which converts the matrix into a column vector.

${ }^{2} \mathrm{~A}$ hollow matrix is a square matrix whose diagonal elements are all equal to zero.
}

totality of incoming flow, and as $\mathcal{P}(\overrightarrow{\mathbf{r}}) \geq \mathcal{A}_{\mathbf{r}}{ }^{-1} \times \mathcal{B}_{\mathbf{r}}$ (componentwise comparison), our centralized optimization problem can be written as:

$$
\begin{array}{ll}
\text { minimize } & f(\overrightarrow{\mathbf{r}})=\omega^{T} \mathcal{A}_{\mathbf{r}}{ }^{-1} \mathcal{B}_{\mathbf{r}} \\
\text { subject to } & \sum_{1 \leq j \leq n} r_{i j}=\sum_{1 \leq j \leq n} r_{j i}, \forall 1 \leq i \leq n ; \\
& \mathcal{A}_{\mathbf{r}}{ }^{-1} \mathcal{B}_{\mathbf{r}} e_{i}^{T} \leq P_{\max }^{i}, \forall 1 \leq i \leq n ;
\end{array}
$$

where $\omega^{T}=\left(\omega_{1}, \omega_{2}, \cdots, \omega_{n}\right)$ is the weight vector for the femto-relays transmit powers (in the simulations we use equal weight for the different powers), and $e_{i}^{T}$ is the transpose of the $i^{\text {th }}$ unit vector in the canonical basis $\left(e_{i}^{T}\right.$ denotes the vector with a 1 in the $i^{\text {th }}$ coordinate and 0's elsewhere.) The minimization of the global power results in a flow conservation constraint: all flows incoming at a given node should be further transmitted (otherwise one could save the power used for bringing this part of the flow to this node), and obviously nothing can be transmitted if it does not reach the node. This flow conservation is used as a first constraint in our problem (first eq. of 5). The second constraint imposes a maximum transmit power at each femto-relay.

Lemma II.1. The centralized-optimization problem in (5) is not convex.

Because of the lack of space, we do not detail the proof of the non-convexity of (5). However this proof turns out to show that the determinant of the Hessian of $f(\overrightarrow{\mathbf{r}})$ is always negative.

\section{THE PROPOSED APPROACH}

In the centralized formulation of the problem, a global knowledge of network parameters and channels is often required. This requirement can be hardly fulfilled in femto-relay networks, as femto-relays act as independent and opportunistic base stations, in order to minimize the network management overheads. Moreover, femto-relays are independent because their installation for residential or enterprise usages is expected to be subject to separate billing, while the opportunistic behavior can be motivated by the attempt of each femto-relay to minimize its own transmit power. Besides, the complexity and the non-convexity of the centralized problem drive us to search for new approaches to model the joint power allocation and multipath routing in femto-relay networks. Our algorithm is composed of two different phases:

- Network partitioning phase, and

- Iterative and distributed formulation phase.

\section{A. Network Partitioning Phase}

Under our assumption that femto-relay networks operate using the time division duplex (TDD) mode, a single frequency band is used for both transmit and receive while assigning alternating time slots to transmit and receive operations. For this aim, an efficient scheduling should be done to decide which nodes should transmit or receive at each time slot. This is of crucial importance in our setting, since only simultaneous transmissions interfere. Our proposition is to partition the set 


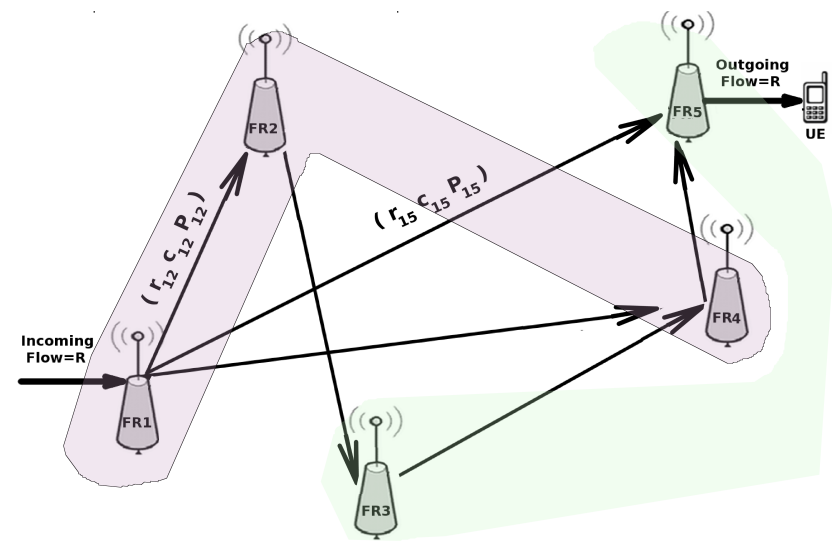

Fig. 2. Interference-based partitioning of 5 femto-relays network.

of $\mathcal{N}$ femto-relays into two clusters based on the interference level. In other terms, we group together the nodes that cause the less interference to each other, and the resulting SINR of the different transmissions is maximized. Such a partition is obtained via a low-complexity centralized optimization problem that takes the expected sum of interference as a partitioning criterion. The problem can be written as follows:

minimize $P(\mathcal{U})=\sum_{l=1}^{k} \sum_{i=1}^{n} \sum_{j=1 ; j \neq i}^{n} u_{i, l} u_{j, l} \mathbb{E}\left(G_{i j}\right) P_{\max }^{i}$

subject to $\sum_{l=1}^{k} u_{i, l}=1, \forall 1 \leq i \leq n$

where:

$$
u_{i, l} \in\{0,1\}, \forall 1 \leq i \leq n, 1 \leq l \leq k
$$

- $\mathcal{U}$ is an $n \times k$ partition matrix, $u_{i, l}$ is a binary variable that is equal to 1 if $N_{i}$ is attributed to the cluster $S_{l}$ and 0 otherwise.

- $\mathbb{E}\left(G_{i j}\right)$ is the expectation of the path gain of link $\ell_{i j}$.

- $d_{i j}$ is the distance between $N_{i}$ and $N_{j}$.

- $k$ is the desired number of clusters (in our case $k=2$ ).

- $n$ is the total number of femto-relays in the network.

Globally, even if this step is centralized, the criterion involves the expectation of the path gains, which depend only on the topology of the femto-relay network (the expectation of the path gain is equal to the path loss $\mathbb{E}\left(G_{i j}\right)=L_{i j}$, and erases the impact of fast fading). Our formulation is thus based on a coordination signal with low rate and scalable signaling that involves very little overhead. Moreover, the above algorithm has a low complexity of $O\left(n^{2} \times k^{2}\right)$. When applied to the network of Figure 1, the above clustering algorithm provides the results depicted in Figure 2.

\section{B. Iterative and distributed formulation phase}

In the second phase, we model the point allocation problem as an iterative and distributed algorithm based on the sequential best response dynamics. Given different time instances $T=\left[\tau_{1}, \tau_{2}, \tau_{3}, \cdots\right]$, each femto-relay $i$ updates its transmit power $J_{i}(\overrightarrow{\mathbf{r}})=\mathcal{A}_{\mathbf{r}}{ }^{-1} \mathcal{B}_{\mathbf{r}} e_{i}^{T}$ at each time $\tau_{k} \in T$ by computing $\overline{\text { Algorithm } 1 \text { Iterative and distributed algorithm based on the }}$ sequential best response dynamics

1: Step 1: Set the initial rate vector $\overrightarrow{\mathbf{r}}_{0}=\overrightarrow{\mathbf{r}}$ such as $\overrightarrow{\mathbf{r}}$ is any matrix in the strategy space $\mathcal{X}$.

2: Step 2: At each time instance $\tau_{k} \in T$, given $\overrightarrow{\mathbf{r}}_{k-1}$, the femto-relays compute, sequentially, their best responses

$$
\mathbf{r}_{i}^{*}\left(\tau_{k}\right) \in \arg \min J_{i}\left(\mathbf{r}_{i}, \mathbf{r}_{-i}\left(\tau_{k-1}\right)\right) ; \forall i \in \Omega
$$

3: Step 3: Repeat Step 2 until $\overrightarrow{\mathbf{r}}\left(\tau_{k}\right)=\overrightarrow{\mathbf{r}}\left(\tau_{k-1}\right)$

its best response as a function of the current SINR levels of other femto-relays in the network. Note that if $J_{i}(\overrightarrow{\mathbf{r}})$ admits multiple minima, we choose randomly one solution from the equivalence class. This algorithm is myopically repeated by the different femto-relays until the equilibrium, if it exists, is reached. At each time, femto-relays have neither memory of past rounds nor speculation of future events. This algorithm can be modeled by the following strategic game:

$$
\mathcal{G}=\left(\Omega,\left(\mathcal{X}_{i}\right)_{i \in \Omega},\left(J_{i}(\overrightarrow{\mathbf{r}})\right)_{i \in \Omega}\right)
$$

where:

- $\Omega$ is the set of $\mathcal{N}$ players (i.e., femto-relays in the network),

- $\mathcal{X}_{i}$ is the set of strategies of the $i^{t h}$ player. It is worth mentioning that as we are restricting the transmission only between nodes belonging to different clusters, the link's rates of the nodes belonging to the same cluster are set to 0 . Hence the strategy set of each player $i \in S_{l} \subset \Omega$ can be expressed by:

$$
\begin{aligned}
& \mathcal{X}_{i}=\left\{\left(r_{i 1}, r_{i 2}, \cdots, r_{i n}\right) \in R_{+}^{n} ; r_{i k}=0, \sum_{j \in \bar{S}_{l}} r_{i j}=\sum_{j \in \bar{S}_{l}} r_{j i},\right. \\
& \left.\mathcal{A}_{\mathbf{r}}{ }^{-1} \mathcal{B}_{\mathbf{r}} e_{i}^{T} \leq P_{\text {max }}^{i}, \forall k \in S_{l}, \forall j \in \bar{S}_{l}\right\}
\end{aligned}
$$

In the following, we refer to the set of pure strategies of all the players by $\mathcal{X} \in R_{+}^{n \times n}$.

- The cost function of each player $i \in S_{l} \subset \Omega$ is expressed by: $J_{i}(\overrightarrow{\mathbf{r}})=\mathcal{A}_{\mathbf{r}}{ }^{-1} \mathcal{B}_{\mathbf{r}} e_{i}^{T}$;

Our proposed iterative and distributed algorithm can thus be seen in Algorithm 1. However, it should be demonstrated that the properties of game (7) always ensure the existence of an equilibrium. For this aim, we refer to theorem of Debreu, Fan and Glicksberg [17] [18].

\section{Theorem III.1. [Debreu-Fan-Glicksberg]}

Let $\mathcal{G}=\left(\Omega,\left(\mathcal{X}_{i}\right)_{i},\left(J_{i}\right)_{i}\right)$ be a strategic form game. If $\forall i \in \Omega$, $\mathcal{X}_{i}$ is a compact and convex set and $J_{i}(x)$ is a continuous function in the profile of strategies $\mathcal{X}$ and quasi-convex in $x_{i}$, then the game $\mathcal{G}$ has at least one pure Nash Equilibrium.

Lemma III.2. The strategic game presented in (7) satisfies the above theorem, hence it admits a pure Nash Equilibrium.

Proof: For simplicity, we prove the applicability of the theorem using a small network composed of $n=3$ femtorelays where $N_{1}$ and $N_{2}$ are transmitting to $N_{3}{ }^{3}$. These

\footnotetext{
${ }^{3}$ As $N_{3}$ is in the receiving mode, its transmit power is set to zero.
} 
findings can also be generalized for any number $n \geq 3$ of nodes. The rate vector $\overrightarrow{\mathbf{r}}$ can be expressed by: $\overrightarrow{\mathbf{r}}=$ $\left(0, r_{12}, r_{13}, r_{21}, 0, r_{23}, r_{31}, r_{32}, 0\right)$, where $r_{12}=r_{21}=0$ since there is no t transmission between $N_{1}$ and $N_{2} ; r_{31}=r_{32}=0$ as $N_{3}$ is in the receiving mode. A necessary and sufficient condition for the existence and positiveness of $\mathcal{P}(\overrightarrow{\mathbf{r}})$ is that:

$$
1-\gamma\left(r_{13}\right) \times \gamma\left(r_{23}\right)>0
$$

where $\gamma\left(r_{i j}\right)=2^{r_{i j}}-1$

Under this condition, one obtains:

$$
\left(\begin{array}{l}
P_{1}(\overrightarrow{\mathbf{r}}) \\
P_{2}(\overrightarrow{\mathbf{r}})
\end{array}\right)=\left(\begin{array}{c}
\frac{1}{1-\gamma\left(r_{13}\right) \times \gamma\left(r_{23}\right)}\left[\frac{\sigma_{3}^{2} \gamma\left(r_{13}\right)}{G_{13}}+\frac{\sigma_{3}^{2} \gamma\left(r_{13}\right) \gamma\left(r_{23}\right)}{G_{13}}\right] \\
\frac{1}{1-\gamma\left(r_{13}\right) \times \gamma\left(r_{23}\right)}\left[\frac{\sigma_{3}^{2} \gamma\left(r_{13}\right) \gamma\left(r_{23}\right)}{G_{23}}+\frac{\sigma_{3}^{2} \gamma\left(r_{23}\right)}{G_{23}}\right]
\end{array}\right)
$$

Observe that, since the original problem in (5) is feasible, then the set $\mathcal{X}$ defined in (8) is compact and convex. Moreover, the cost functions of $N_{1}$ and $N_{2}$ (i.e., $P_{1}(\overrightarrow{\mathbf{r}})$ and $P_{2}(\overrightarrow{\mathbf{r}})$ ) are strictly convex in $\mathcal{X}_{1}$ and $\mathcal{X}_{2}$, respectively. In fact, we have:

$$
\begin{aligned}
& \quad \frac{d^{2} P_{1}(\overrightarrow{\mathbf{r}})}{d r_{13}^{2}}=\frac{(\ln (2))^{2}}{\left(1-\gamma\left(r_{13}\right) \gamma\left(r_{23}\right)\right)^{3}}\left[\sigma_{3}^{2} \gamma\left(r_{13}\right) \gamma\left(r_{23}\right) \frac{1}{G_{13}}+\frac{\sigma_{3}^{2} \gamma\left(r_{13}\right)}{G_{13}}+\right. \\
& \left.\gamma\left(r_{13}\right) \gamma\left(r_{23}\right) \frac{\sigma_{3}^{2} \gamma\left(r_{13}\right)}{G_{13}}+\sigma_{3}^{2} \gamma\left(r_{13}\right) \gamma\left(r_{23}\right) \frac{1}{G_{13}}\right] \geq 0, \text { and } \\
& \quad \frac{d^{2} P_{2}(\overrightarrow{\mathbf{r}})}{d r_{23}^{2}}=\frac{(\ln (2))^{2}}{\left(1-\gamma\left(r_{13}\right) \gamma\left(r_{23}\right)\right)^{3}}\left[\sigma_{3}^{2} \gamma\left(r_{13}\right) \gamma\left(r_{23}\right) \frac{1}{G_{23}}+\frac{\sigma_{3}^{2} \gamma\left(r_{23}\right)}{G_{23}}+\right. \\
& \left.\gamma\left(r_{13}\right) \gamma\left(r_{23}\right) \frac{\sigma_{3}^{2} \gamma\left(r_{23}\right)}{G_{23}}+\sigma_{3}^{2} \gamma\left(r_{13}\right) \gamma\left(r_{23}\right) \frac{1}{G_{23}}\right] \geq 0 .
\end{aligned}
$$

Hence our strategic game admits a Nash equilibrium that can be reached using Algorithm 1.

When applied to the network of Figure 1, one obtains at the equilibrium the femto-relays' transmit powers as well as the corresponding multi-path routing, as shown in Figure 3. It is worth mentioning that the links' rates and capacities are expressed in bit/s/Hz while the links' powers are in $\mathrm{mW}$.

\section{PERformance Evaluation}

The performance of the proposed approach is evaluated below in terms of power reduction w.r.t. classical routing techniques, namely direct transmission and shortest path:

- In the direct transmission technique, the data are sent directly from the source (e.g., the closest femto-relay to the macrocell) to the destination (i.e., the user equipment) using one-hop communication. This is possible as we are considering that all femto-relays are within the coverage of each other. Intuitively, a strategic game formulation for this type of communication is not required as there is only one femto-relay (i.e., one player) that transmits the data to the destination.

- In the shortest path technique, the best route from source to destination through a shortest path selection function (e.g., Dijkstra or Bellman Ford algorithm) is selected. Intuitively, this technique does not support multi-path communications, as there is a single route between source and destination. Hence, this technique can be seen as a power allocation with no multi-path routing.

We first illustrate the convergence of our approach to the Nash equilibrium within a finite number of iterations. We simulate several scenarios with a dense network of 200 femtorelays uniformly distributed in a $300 m \times 300 m$ area. The

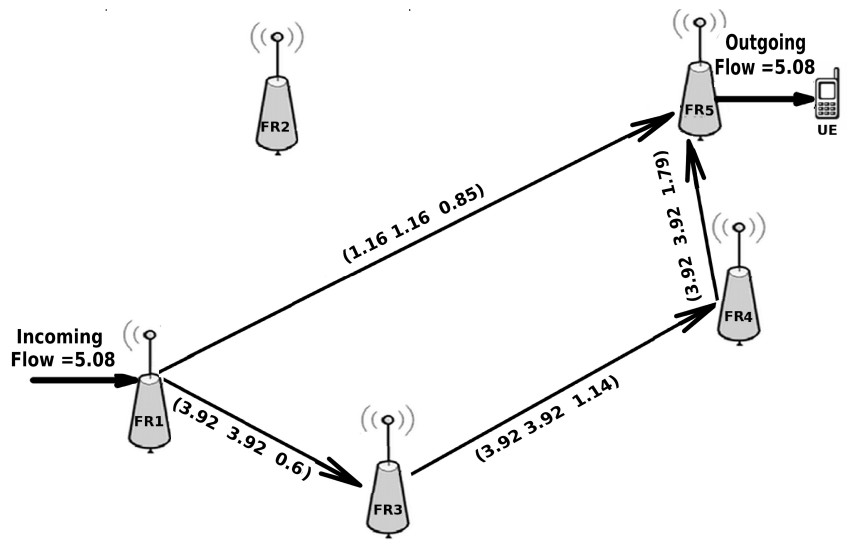

Fig. 3. The transmit powers and the corresponding multi-path routing at the equilibrium of Algorithm 1 for the 5 femto-relays network

maximum power of each femto-relay is set to $15 \mathrm{dBm}(31.61$ $\mathrm{mW}$ ), and the AWGN level at the receiver to $5 \mathrm{~dB}$.

It is worth mentioning that the network is not able to afford any value of the flow because it is always limited by the maximum power thresholds (i.e., in some cases, a given node, even if it uses its maximum power, is not able to send the totality of the incoming flow). To tackle this issue, we define the maximum affordable data flow $R_{\max }$ in the network as the minimum of the various nodes capacities in their extreme cases: $R_{\max }=\min \left(C_{i}\right), \forall i$ where $C_{i}$ is the capacity of Node $i$ in its extreme case that happens where $N_{i}$ works at its maximum power and suffers from the highest level of interference (when all the nodes in the network work at their maximum transmit power level). An intensive simulation campaign indicates that, provided that the incoming flow rate does not exceed $R_{\max }$, the proposed algorithm converges with overwhelming probability.

\section{A. Convergence to equilibrium}

In order to study the convergence rate of our algorithm, we take the case of an incoming flow $R$ that is equal to $80 \%$ of $R_{\max }$. Figure 4 shows the variation of the total femtorelays transmit power in the network $\left(\sum_{i} P_{i}, \forall i \in \Omega\right)$ as a function of two randomly chosen initial states $\overrightarrow{\mathbf{r}}_{0}$ and $\overrightarrow{\mathbf{r}}_{0} \in \mathcal{X}$ and as a function of the number of rounds where each round refers to the change of one player's action. The red and green lines in the figure refer to the variation of the total consumed power for the two different initial states where the dotted line represents the power at the equilibrium of the game. Our algorithm clearly converges to the Nash Equilibrium within a finite number of rounds. The convergence is also very fast (a femto-relay updates its power only once before convergence) and scalable since the speed of convergence is on average equal to $O(1 \times n)$.

\section{B. Power reduction}

Fig. 5 illustrates the power reduction of our proposed approach with respect to direct transmission and shortest path routing. The results show clearly that our joint power allocation and multi-path routing algorithm ensures important 


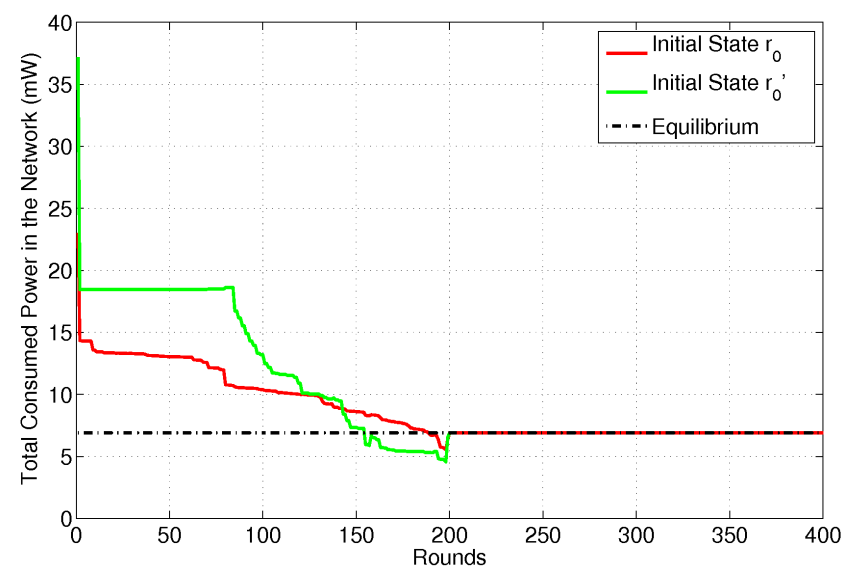

Fig. 4. Convergence rate of the proposed approach as a function of the number of rounds for a network of 200 femto-relays. The convergence is fast (each femto-relay updates its power once before convergence) and scalable (the rate of convergence is on average equal to $O(1 \times n)$ )

improvements in terms of power reduction with respect to the direct transmission and the shortest path that can be seen as power allocation techniques that do not support the multi-path routing. As a matter of fact, the total consumed power of our approach is divided by about 6 and 3 when respectively compared to the direct transmission and shortest path techniques.

\section{CONCLUSION}

One of the key contributions of this paper is to propose a procedure to perform power allocation and routing in a joint manner while works available in the literature provide power allocation schemes for a fixed routing scheme. The proposed approach allows to minimize the total consumed power of femto-relay networks, operating over TDD mode, while guaranteeing a QoS constraint in terms of transmission rate. The proposed algorithm has several very attractive features. It relies on local information and low-rate and scalable signaling. Simulations show that it converges with overwhelming probability and rapidly (one iteration per femto-relay usually suffices to get the convergence to equilibrium). Implementing the proposed algorithm yields very significant gains in terms of total power consumption. Based on in these results, several extensions might be explored, e.g., using other performance criteria such as energy-efficiency (in bit per Joule) appears as an interesting complementary approach to be studied.

\section{ACKNOWLEDGMENT}

This work was partially funded by Orange Labs.

\section{REFERENCES}

[1] I. F. Akyildiz, D. M. Gutierrez-Estevez, E. Chavarria-Reyes "Femtorelay systems and methods of managing same" US Patent Application, No. 20120076027, Pub. Mar. 29, 2012.

[2] I. F. Akyildiz, E. Chavarria-Reyes, D. M. Gutierrez-Estevez, R. Balakrishnan and J. R. Krier "Enabling next generation small cells through femtorelays " Physical Communication, Vol.9, pp. 1-15, 2013.

[3] D. Lopez-Perez , A. Valcarce, G. de la Roche, J. Zhang, "OFDMA femtocells: A roadmap on interference avoidance" IEEE Comm. Magazine, Vol.47, No. 9, pp. 41-48, Sept. 2009.

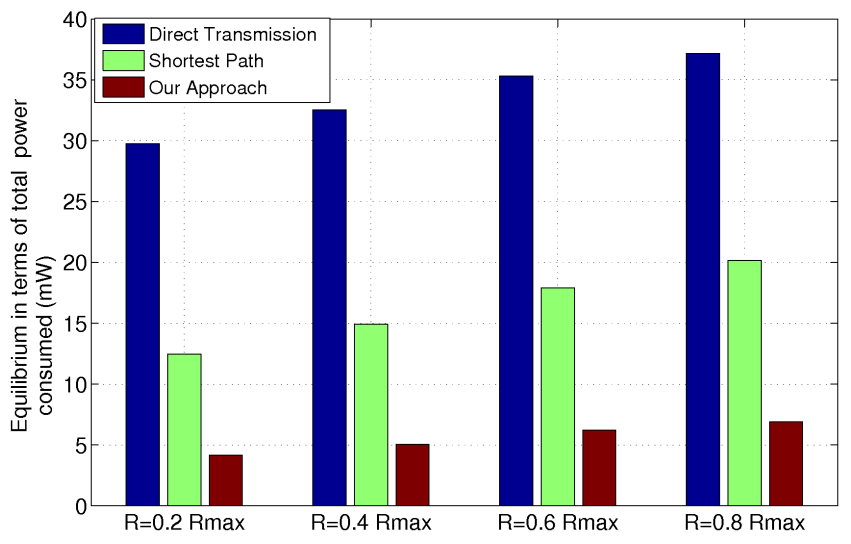

Fig. 5. Comparison of the sum of femto-relays transmit power for the various approaches: direct transmission, shortest path and our proposed approach as a function of different incoming flow values.

[4] S. Hoteit, S. Secci, R. Langar, G. Pujolle and R. Boutaba "Bankruptcy Game Approach for Resource Allocation in Cooperative Femtocell Networks" in Proc. of IEEE Global Communications Conference (IEEE GLOBECOM), Anaheim, CA, USA, 2012.

[5] R. Ghaffar, H. Pin-Han, "Cross-tier interference mitigation in Femtomacro cellular architecture in downlink" in Proc. of IEEE International Conference on Communications (IEEE ICC), Budapest, 2013.

[6] T. Zahir, K. Arshad, A. Nakata, K. Moessner, "Interference Management in Femtocells," IEEE Communications Surveys and Tutorials, vol.15, no.1, pp.293-311, 2013.

[7] X. Xu, Y. Li, R. Gau, X. Tao "Joint Voronoi diagram and game theory-based power control scheme for the HetNet small cell networks", EURASIP Journal on Wireless Com. and Network.,vol. 2014, no. 213, 2014.

[8] P. Gérold, T. T. Ngoc Pham, F. Alberge, P. Duhamel "Multi-Hop, MultiRoute Power Minimisation in Ad-Hoc Network, in Proc. of IEEE Int. Conf. on Acoustics, Speech and Sig. Proc. (ICASSP), Kyoto, Japan, 2012.

[9] X. Kang; R. Zhang; M. Motani, "Price-Based Resource Allocation for Spectrum-Sharing Femtocell Networks: A Stackelberg Game Approach", IEEE J. on Selected Areas in Com., Vol. 30, No. 3, pp. 538-549, 2012.

[10] I. Guvenc, M.R. Jeong, F. Watanabe, H. Inamura, "A hybrid frequency assignment for femtocells and coverage area analysis for co-channel operation", IEEE Com. Letters, Vol.12, No.12, pp.880-882, 2008.

[11] H.S. Jo, C. Mun, J. Moon, J.G. Yook "Interference Mitigation Using Uplink Power Control for Two-tier Femtocell Networks", IEEE Transactions on Wireless Communications, Vol. 8, No. 10, pp. 4906-4910, 2009.

[12] E. J. Hong, S. Y. Yun, D. H. Cho "Decentralized Power Control Scheme in Femtocell Networks: A Game Theoretic Approach", Int. Symp. on Personal, Indoor and Mobile Radio Communications PIMRC, 2009.

[13] J.S. Pang, G. Scutari, F. Facchinei, C. Wang "Distributed Power Allocation With Rate Constraints in Gaussian Parallel Interference Channels", IEEE Transactions on in Info. Theory, vol.54, no.8, pp.3471-3489, 2008.

[14] F. Pantisano, K. Ghaboosi, M. Bennis, M. Latva-Aho, "Interference avoidance via resource scheduling in TDD underlay femtocells" in Proc. of IEEE International Symposium on Personal, Indoor and Mobile Radio Communications Workshops (PIMRC Workshops), 2010.

[15] D. WINNER II, "Winner II Channel models", IST-4-027756 WINNER II, D1.1.2 V1.2, Tech. Rep., Sept. 2007.

[16] D. Xu, F. Hu, Q. Wang, Z Niu "A low-complexity power allocation scheme for distributed wireless links in Rayleigh fading channels with capacity optimization" in Proc. of International Conference on Mobile Ad-hoc and Sensor Networks, Wuhan, China, 2005.

[17] D. Fudenberg, J. Tirole, "Game Theory" The MIT Press., 1991.

[18] S. Lasaulce and H. Tembiné, "Game Theory and Learning for Wireless Networks: Fundamentals and Applications" Academic Press, Elsevier, pp. 1-336, Aug. 2011, ISBN 978-0123846983. 